Eur. J. Clin. Chem. Clin. Biochem.

Vol. 31, 1993, pp. 29-33

(C) 1993 Walter de Gruyter \& Co.

Berlin - New York

\title{
New Approaches with the Chem-1 Creatinine Determination
}

\author{
By B. G. Blijenberg, L. Zwang and M. van Tilborg \\ Academic Hospital Rotterdam-Dijkzigt, Department of Clinical Chemistry, Rotterdam, The Netherlands
}

(Received June 22/August 17, 1992)

Summary: Three modifications of the Chem-1 determination of serum creatinine were tested. Two different algorithms developed for compensating the interference of bilirubin were compared with the current one by analysing bilirubin-enriched albumin solutions and 82 icteric serum samples. A number of known other interfering substances were also tested.

The modification involving parallel bichromatic measurement plus bilirubin correction appeared to give the best performance.

However, despite the improvements, the determination is not yet completely satisfactory.

\section{Introduction}

In a previous study we discussed the problems related to the determination of creatinine in icteric specimens (1). That study was undertaken because of doubt concerning some of our Chem-1 results. We also mentioned the involvement of the Bayer-Technicon company, which manufactures the Chem-1 analyser. In this article we present the results of performance studies of three modifications of the creatinine assay, two of them being experimental. All methods are kinetic Jaffe methods. The original, unmodified procedure is based on incubation of the sample with picric acid and initiation of the colour reaction with sodium hydroxide; there is no mathematical correction algorithm for bilirubin.

The modifications differ in two respects from this procedure. First, the addition of picric acid and sodium hydroxide is reversed. Second, two different bilirubin correction algorithms are applied, i.e. a selective bichromatic correction algorithm, and a parallel bichromatic measurement with a bilirubin correction for icteric samples.

We also studied the influence of other well-known interfering factors.

\section{Materials and Methods}

Materials

Creatinine, unconjugated bilirubin, glucose, $\beta$-hydroxybutyric acid, ascorbic acid and acetone were obtained from Merck (Germany), conjugated bilirubin (ditaurate, di Na) from Porphyrin Products Inc. (Logan, Utah, U.S. A.) and human albumin from Behring Werke (Germany).

The following drugs were obtained from the Department of Pharmacy in our hospital:

$$
\begin{aligned}
& \text { 1. cephalosporins - cephotaxin- } \mathrm{Na}\left(\text { Claforan }^{\circledR}\right) \\
& \text { - cephalotine-Na (Keflin }{ }^{(D)} \\
& \text { - cephuroxim-Na (Zinacef }{ }^{\boxplus} \text { ) }
\end{aligned}
$$

2. dopamine- $\mathrm{HCl}$

3. dobutamine- $\mathrm{HCl}$ (Dobutrex ${ }^{(1)}$ )

Icteric serum samples were provided by various departments in our hospital. Only samples with a total bilirubin concentration higher than $100 \mu \mathrm{mol} / \mathrm{l}$ were used. They were stored at $-80^{\circ} \mathrm{C}$ prior to analysis. Table 1 shows the frequency distribution of the bilirubin concentrations. Cyclosporin-containing serum samples were obtained from our transplantation unit. All cyclosporin concentrations, as determined by radioimmunoassay, were lower than $100 \mathrm{mg} / \mathrm{l}$ which is considered to be therapeutic.

\section{Methods}

An HPLC-based method already described by us (2) was used as the reference method. 
Tab. 1. Frequency distribution of the total bilirubin concentrations

\begin{tabular}{ll}
\hline $\begin{array}{l}\text { Concentration range } \\
(\mu \mathrm{mol} / \mathrm{l})\end{array}$ & Number of samples \\
\hline $101-150$ & 17 \\
$151-200$ & 20 \\
$201-250$ & 12 \\
$251-300$ & 6 \\
$301-350$ & 2 \\
$351-400$ & 6 \\
$401-450$ & 6 \\
$451-500$ & 6 \\
$>500$ & 7 \\
\hline
\end{tabular}

We applied the following creatinine modifications of the creatinine assay to the Chem-1.

1. Version 1 . This version is commercially available. The sample is mixed with picric acid and incubated for 5 minutes, after which sodium hydroxide is added to initiate the reaction. The $\Delta \mathrm{A}$ between 30 and 90 seconds is measured at $500 \mathrm{~nm}$, and is proportional to the creatinine concentration.

2. Version 2. This version is described by Levy et al. (3). It differs from version 1 mainly in the reversal of the order of addition of the reagents, and in the measuring and calculation principle. Absorbance is measured at 500 and $600 \mathrm{~nm}$ after addition of sodium hydroxide to the sample. The values reflect the bilirubin concentration, and if they exceed a certain threshold, a ratio test is performed for correction of the final result.

3. Version 3. Here the same chemistry is used as in version 2. A parallel bichromatic absorbance measurement at 500 and $600 \mathrm{~nm}$ is performed. The bilirubin correction is based on the difference between the absorbances at 500 and $600 \mathrm{~nm}$ in the first flow cell.

For all modifications, the Chem-1 was calibrated with the Setpoint Chem-1 Calibrator. The system specific value was established for this calibrator with our HPLC method by running a sample on five consecutive days in triplicate. The slope of the Chem-1 was adjusted to 1.0 and the intercept to $0 \mu \mathrm{mol} / \mathrm{l}$.

The interference study was performed according to the CERMAB schedule (4). A stock solution of drugs, metabolites, haemoglobin and lipids (Intralipid ${ }^{(5)}$ ) in water was diluted with human albumin solution $(40 \mathrm{~g} / \mathrm{l})$ to give $5-7$ dilutions. The dilutions were chosen to give low, normal, therapeutic or elevated levels.

Unconjugated and conjugated bilirubin were used in accordance with 1.c. $(5,6)$.

\section{Results}

\section{Bilirubin in vitro and in vivo}

We started the study by comparing all three Chem- 1 creatinine versions using bilirubin-enriched albumin solutions. Three aqueous creatinine standard solutions were compared, containing about 100, 300 and $500 \mu \mathrm{mol} / 1$. The results for $100 \mu \mathrm{mol} / 1$ are shown in figure 1 . The other two concentrations gave a similar slope.

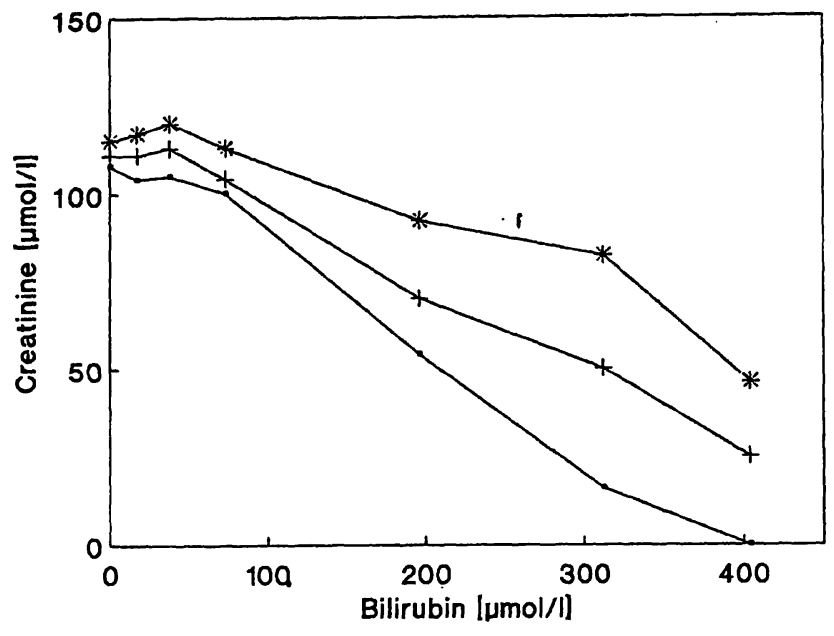

Fig. 1. The influence of unconjugated bilirubin (added as bilirubin-enriched albumin solutions) on three Chem-1 creatinine modifications:

$\mathrm{Q}=$ version $1=$ original version

$t=$ version $2=$ modification Levy

$*=$ version $3=$ research version

The procedure was repeated using ditaurobilirubinenriched albumin solutions (2). The results can be seen in figure 2.

From figures 1 and 2 it is clear that version 3 gives the best performance, although a bilirubin effect can still be measured. Nevertheless, it seemed worthwhile to test the method modifications in the daily routine by analysing icteric patient specimens. We therefore analysed 82 icteric sera, with total bilirubin concentrations ranging from 104 to $570 \mu \mathrm{mol} / 1$ (the distribution is given in table 1), using all three versions of the method. The results are presented graphically in figures 3,4 and 5 .

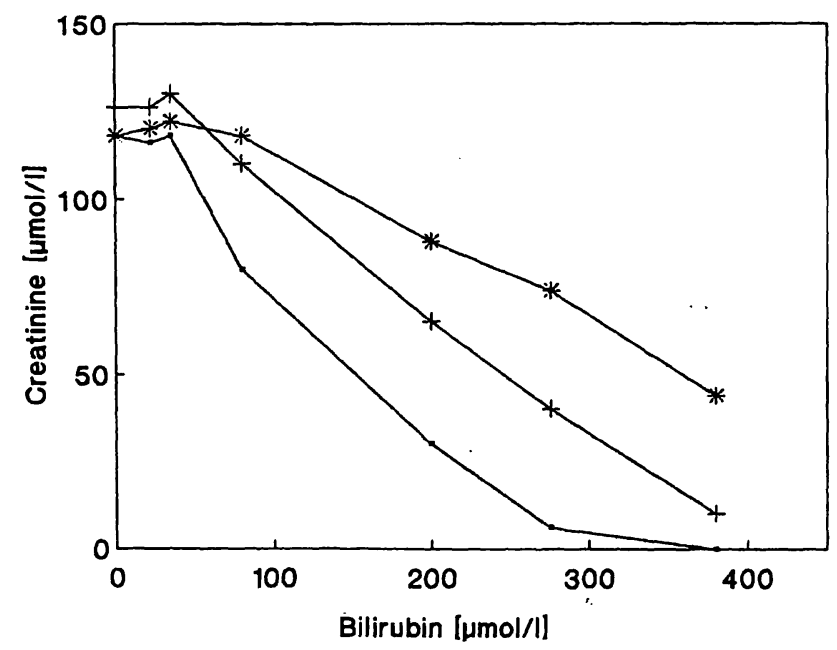

Fig. 2. The influence of ditaurobilirubin (added as ditaurobilirubin-enriched albumin solutions) on three Chem-1 creatinine modifications:

$\square=$ version $1=$ original vèrsion

$t=$ version $2=$ modification Levy

* $=$ version $3=$ research version 


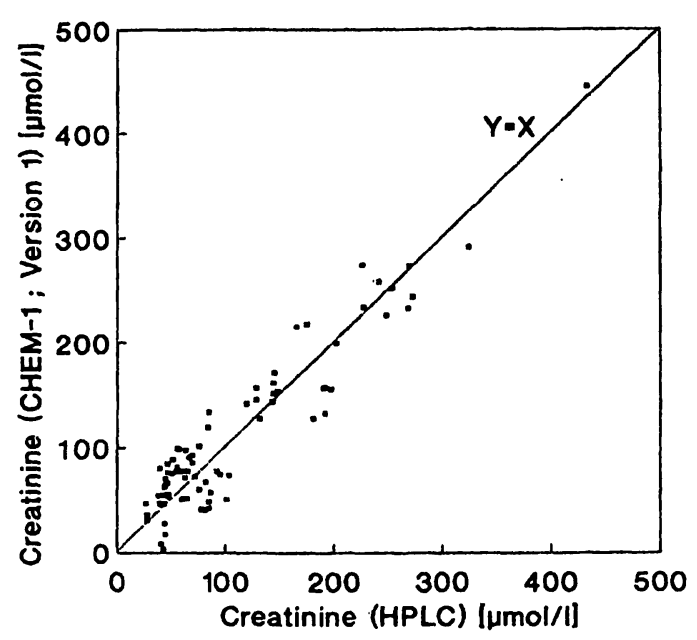

Fig. 3. Comparison of the Chem-1 version 1 creatinine formulation (y-axis) and our HPLC-based reference method ( $x$-axis) with 82 icteric samples.

The line $y=x$ is also depicted.

Bablok and Passing statistics: $y=1.01 x+8 ; r=0.94$

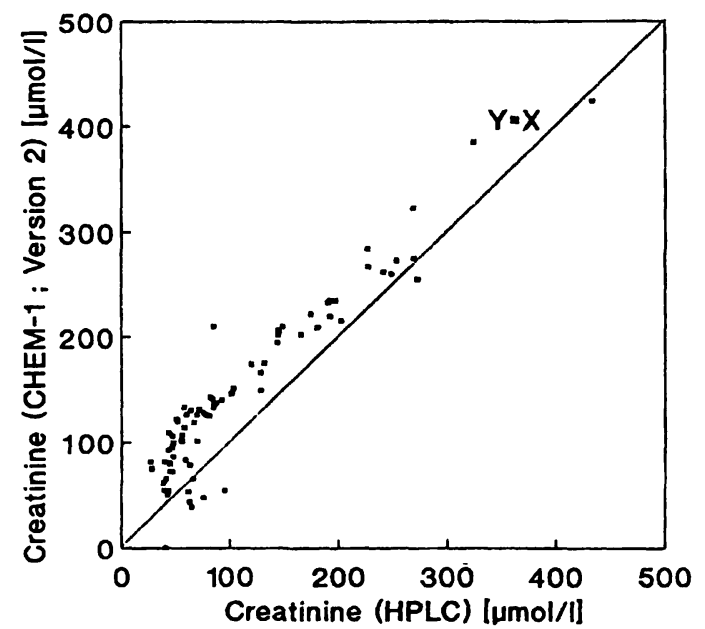

Fig. 4. Comparison of the Chem-1 version 2 creatinine formulation ( $y$-axis) and our HPLC-based reference method (x-axis) with 82 icteric samples.

The line $\mathbf{y}=\mathbf{x}$ is also depicted.

Bablok and Passing statistics: $\mathrm{y}=1.06 \mathrm{x}+40 ; \mathrm{r}=0.94$

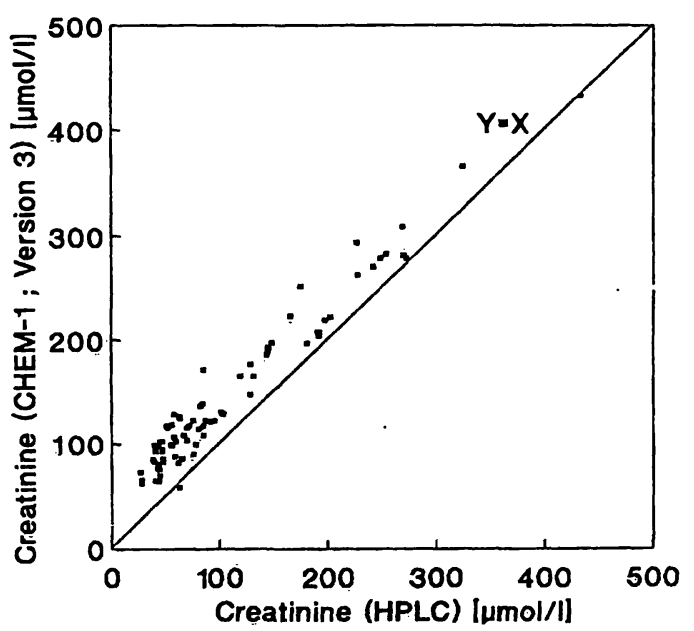

Fig. 5. Comparison of the Chem-1 version 3 creatinine formulation (y-axis) and our HPLC-based reference method ( $x$-axis) with 82 icteric samples.

The line $y=x$ is also depicted.

Bablok and Passing statistics: $y=0.97 x+40 ; r=0.98$
It is clear that the in vivo results, like the in vitro results, also showed improvements. On the other hand, it is also obvious that the final goal of acceptable accuracy for all specimens is not attained by version 3 . This is best illustrated by the alternative presentation of results shown in figures 6 and 7.

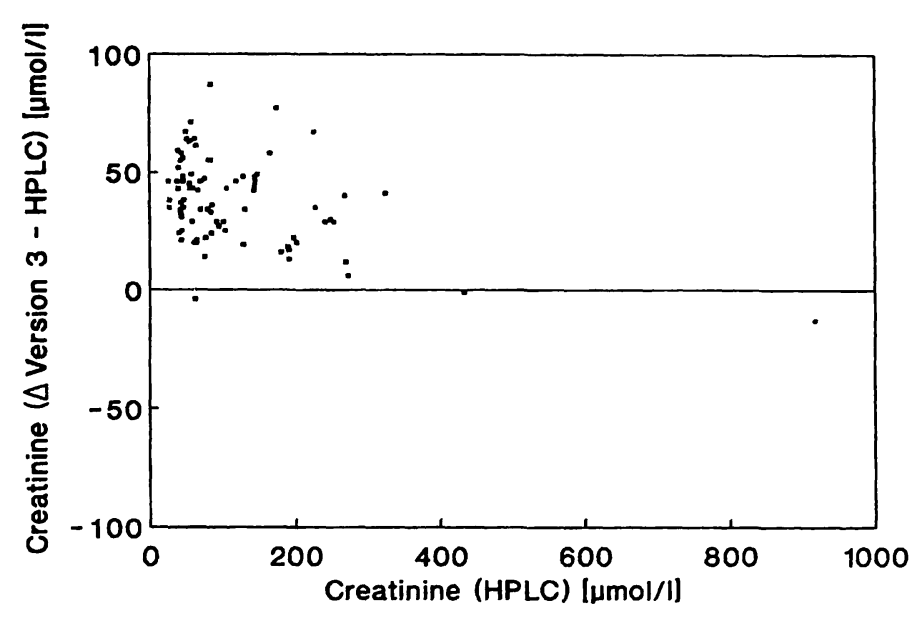

Fig. 6. The difference in creatinine values between version 3 and HPLC (y-axis) plotted against the creatinine concentrations (x-axis) of the 82 icteric samples.

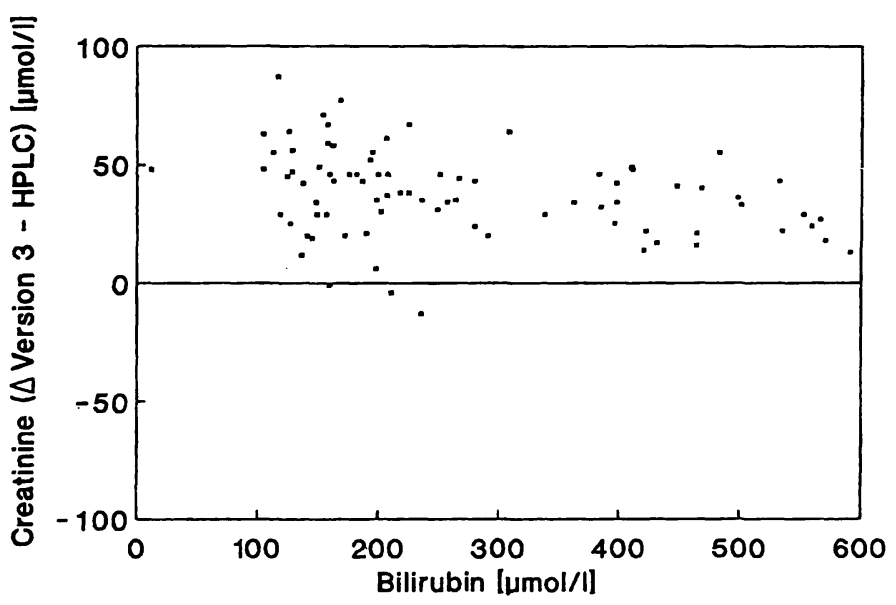

Fig. 7. The difference in creatinine values between version 3 and HPLC ( $y$-axis) plotted against the bilirubin concentrations (x-axis) of the 82 icteric samples.

\section{Other interferents in vitro}

Bilirubin is not the only interfering substance in the creatinine determination. Despite the efforts to improve the Chem-1 creatinine methodology, we also felt the need to test other well-known interfering compounds. We therefore investigated the effects of haemoglobin, lipids, drugs (ascorbic acid, dopamine, dobutamine and cephalosporins) and carbohydrate/lipid metabolites (glucose, acetone and $\beta$-hydroxybutyric acid).

In all these studies we used the same system, i.e. human albumin-based creatinine standards of about 100,300 and $300 \mu \mathrm{mol} / \mathrm{l}$ with increasing amounts of 
interferents. The tested concentrations of substances that normally occur in vivo ranged from about normal or zero values up to highly pathological. The tested concentrations of drugs ranged from subtherapeutic to severely elevated levels according to the information sheets of the manufacturers of the drugs. A survey of all the results is given in table 2 .

Tab. 2. Some interference data on all Chem-1 creatinine modifications

\begin{tabular}{llll}
\hline Constituent & Version 1 & Version 2 & Version 3 \\
\hline Bilirubin (unconj.) $\left.)^{1}\right)$ & + & + & \pm \\
Bilirubin (conj.) & + & + & \pm \\
Haemoglobin & \pm & \pm & \pm \\
Lipids & $\pm^{2}$ ) & $\pm^{2}$ ) & $\pm^{2}$ ) \\
Glucose & $\pm^{3}$ ) & $\pm^{3}$ ) & $\pm^{3}$ ) \\
Acetone & - & - & - \\
B-Hydroxy butyric acid & - & - & - \\
Ascorbic acid & - & - & - \\
Cephotaxim & \pm & \pm & \pm \\
Cephalotine $::$ & - & - & - \\
Cephuroxim & - & - & - \\
Dobutamine & - & - & - \\
Dopamine & + & \pm & \pm \\
Cyclosporin & - & - & -4 \\
\hline
\end{tabular}

$+=$ influence

$\pm=$ moderate influence

$-=$ no influence

1) $=$ see figures 1 and 2

2) = moderate at elevated concentrations

$\left.{ }^{3}\right)$ = moderate at glucose concentrations higher than $40 \mathrm{mmol} / \mathrm{l}$

$\left.{ }^{4}\right)$ see figure 8

\section{Cyclosporin in vivo}

The effect of cyclosporin was studied in vivo.

Figure 8 shows the results of the analyses with version 3 and by HPLC.

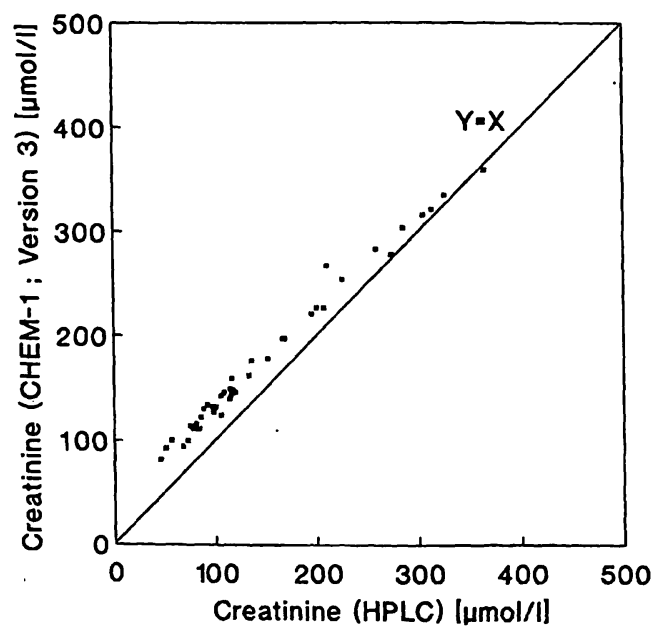

Fig. 8. Comparison of the Chem-1 version 3 creatinine formulation ( $y$-axis) and our HPLC based reference method (x-axis) with 66 cyclosporin-containing serum samples.

The line $\mathrm{y}=\mathrm{x}$ is also depicted.

Bablok and Passing statistics: $\mathrm{y}=0.94 \mathrm{x}+41 ; \mathrm{r}=0.99$

\section{Discussion}

For a better understanding it is helpful to show the performance of version 3 with non-icteric, non-haemolytic and non-lipaemic samples. A graph is given in figure 9.

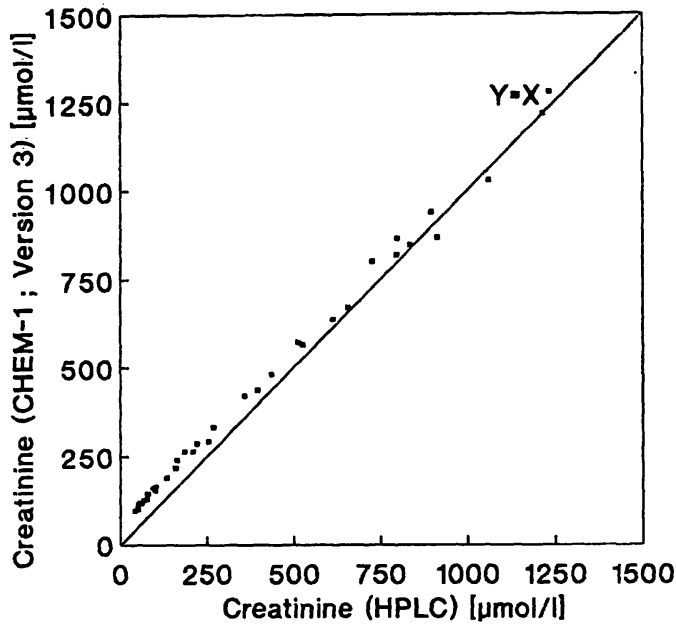

Fig. 9. Comparison of the Chem-1 version 3 creatinine formulation (y-axis) and our HPLC-based reference method (x-axis) with 36 non-icteric, non-haemolytic and non-lipaemic serum samples.

The line $\mathrm{y}=\mathrm{x}$ is also depicted.

Bablok and Passing statistics: $y=0.96 x+62 ; r=1.00$

Versions 1 and 2 show comparable performances, and their results are therefore not depicted.

The results shown in figure 9 are acceptable. The same holds true for the precision of the measurement. We found a coefficient of variation of $2.5 \%$ at 140 $\mu \mathrm{mol} / \mathrm{l}$ and $1.5 \%$ at $750 \mu \mathrm{mol} / 1$ (10 days).

This study confirms our earlier findings (1), i.e. a change in the reagent formulation and/or change in the method of calculation can influence the results substantially. In this respect, as can be seen from figures 3, 4 and 5, version 3 shows the best performance with our set of samples. This is also obvious from the standard error about the regression line $\left(s_{\mathrm{y} / \mathrm{x}}\right)$. For version 1 we found an $s_{y / x}$ of $19.4 \mu \mathrm{mol} / \mathrm{l}$; the corresponding values for versions 2 and 3 were 20.1 and $12.1 \mu \mathrm{mol} / 1$, respectively. The same range $(0-500$ $\mu \mathrm{mol} / \mathrm{l})$ taken from the samples depicted in figure 9 $(n=23)$ gave an $\mathrm{s}_{\mathrm{y} / \mathrm{x}}=6.2 \mu \mathrm{mol} / \mathrm{l}$.

Comparison of figures 5 and 9 gives the impression that the results obtained with icteric specimens approach those obtained with non-icteric specimens, except that the first data show a larger scatter. This raises the question, in relation to the bilirubin interference, of the limitation of the kinetic Jaffe procedure as chosen by Bayer-Technicon: We wonder if there is any more to be gained by further attempted improve- 
ment. Some "fine-tuning" is possible, as can be seen from figure 7 , and this is qualitatively confirmed by the results shown in figures 1 and 2 . Of course, care has to be taken in comparing figures 1,2 and 7 because of possible matrix effects (artificial samples vs. patient samples). It is difficult to compare the new findings with other comparable techniques. Based on our own experience, partly described in our previous publication, we feel that the results obtained with icteric serum samples are not better and not worse than those other techniques. Figure 5 probably shows more or less the state of the art for a kinetic Jaffe method.

The comparison of all graphs is somewhat masked by our calibration of the Chem-1. Using the manufacturer's system-specific value of the Chem-1 calibrator and applying an intercept of $44 \mu \mathrm{mol} / 1$, also stated by the manufacturer, the patient results are assumed to be accurate. Since we had reason to question this calibration system (7), we decided to establish our own values (see Materials and Methods). This resulted in a different calibrator value and a different intercept and slope. It certainly means that the differences between the measured results and the HPLCvalues (see for instance figures 6 and 7) are actually less pronounced. It may also be worthwhile to investigate whether the matrix of the Chem-1 calibrator is appropriate.

The various data also show that bilirubin is not the only interferent. We checked the drug content of a number of serum samples. Most of the information thus obtained was difficult to interpret, because in all cases the samples contained three or even more drugs. On the other hand, the in vitro drug interference study does not present a negative picture, although we are aware that our choice was limited. For example, we investigated the effects of three cephalosporins that are widely used in our hospital, but more are available. Of course, the ideal but also the most laborious way of studying the influence of drugs is the in vivo model that we used for the cyclosporins. It is clear from figure 8 that version 3 performs well.

Taking all measurements and all consideration into account, it is obvious that, despite the improvements made, we cannot be completely satisfied with the results, especially those in the lower and normal range. There still is too much doubt concerning the accuracy of values. We experienced most problems with critical care patients (transplantation patients, neonates), where a number, sometimes many, creatinine requests were made over a relatively long period.

We think that version 3 can be followed by version 4 (fine-tuning of the bilirubin correction algorithm and maybe an improved calibration procedure), but even then there will be doubt because of other interferents. We may need a completely different approach for creatinine.

\section{Acknowledgement}

Many thanks are due to Drs. J. Levine and E. Szymanski (Bayer-Technicon, Tarrytown, U.S. A.) for practical support (software and reagents) and stimulating discussions. Analytical support was given by $R$. J. Leeneman, $C$. J. M. van Leeuwen and $L$.J. Perret and clerical assistance by A. P. Copper-Staamer. The help of Dr. C. J. Hesse (Department of Internal Medicine) for providing the cyclosporin-containing patient samples and of Dr. J.W. Meilink (Department of Pharmacy) for providing the drugs is greatly acknowledged.

\title{
References
}

1. Blijenberg, B. G., Liesting, E. C. \& Zwang, L. (1992) Creatinine and automatic analysers in relation to icteric specimens. Eur. J. Clin. Chem. Clin. Biochem. 30, 779-784.

2. Zwang, L. \& Blijenberg, B. G. (1991) Assessment of a selected method for creatinine with special emphasis on bilirubin interference. Eur. J. Clin. Chem. Clin. Biochem. $29,795-800$.

3. Levy, S., Rutman, M. \& Shihabi, Z. (1990) A novel bilirubin interference correction technique applied to the Technicon Chem-1. Clin. Chem. 36, 1113-1114.

4. Société Française de Biologie Clinique. Commission Validation de Techniques - Protocol de validation de techniques (1986) Ann. Biol. Clin. 44, 686-745.

5. Doumas, B. T., Wu, T. W., Poon, K. C. P. \& Kendrzejczak, B. (1985) Chemical nature of a synthetic bilirubin conjugate and its reactivities in the total and direct reactions by the Jendrassik-Grof method. Clin. Chem. 31, 1677-1682.

6. Franzini, C., Morelli, A. M. \& Cattozzo, G. (1991) Use of a synthetic soluble bilirubin derivative to assess interference in creatinine measurements. Clin. Chem. 37, 236-238.

7. Van Suijlen, J. D. E., Blijenberg, B. G., Boerma, G. J. M. \& Leijnse, B. (1991) Calibration of Technicon Chem-1 multitest analysers. J. Clin. Chem. Clin. Biochem. 29, 205-208.

\author{
Dr. B. G. Blijenberg \\ Department of Clinical Chemistry \\ Academic Hospital Rotterdam-Dijkzigt \\ Dr. Molewaterplein 40 \\ NL-3015 GD Rotterdam \\ The Netherlands
}


。 